\title{
AN EXAMPLE OF A NON-EXPOSED EXTREME FUNCTION IN THE UNIT BALL OF $H^{1}$
}

\author{
by JYUNJI INOUE*
}

(Received 12th May 1992)

Dedicated to professor Mitsuru Nakai on his sixtieth birthday

\begin{abstract}
We construct a non-exposed extreme function $f$ of the unit ball of $H^{1}$, the classical Hardy space on the unit disc of the plane, which has the property: $f(z) /(1-q(z))^{2} \notin H^{1}$ for any nonconstant inner function $q(z)$. This function constitutes a counterexample to a conjecture in D. Sarason [7].
\end{abstract}

1991 Mathematics subject classification: Primary 30 D 55, 46 J 15; secondary 46 B 20.

Let $D$ be the open unit disc in the complex plane $C$, with boundary $T=\partial D$. For $1 \leqq p \leqq \infty, L^{p}=L^{p}(T)$ denotes the Lebesgue space on $T$, and $H^{p}=H^{p}(D)$ the Hardy space on $D$. Occasionally, we identify $f \in H^{1}(D)$ with its boundary function $f\left(e^{i t}\right)$ on $T$. For $\phi \in L^{q}(1 / p+1 / q=1), L=L_{\phi}$ denotes a bounded linear functional on $H^{p}$ defined by

$$
L(f)=\int_{0}^{2 \pi} f\left(e^{i t}\right) \phi\left(e^{i t}\right) d t / 2 \pi
$$

with the norm $\|L\|=\sup \left\{|L(f)|: f \in H^{p},\|f\|_{p} \leqq 1\right\}$. If $L$ is nonzero, we put $S_{L}=$ $\left\{f \in H^{p}: L(f)=\|L\|,\|f\|_{p} \leqq 1\right\}$. $S_{L}$ is the solution set of a well-known linear extremal problem in $H^{p}$.

When $1<p \leqq \infty$, the structure of the set $S_{L}$ is simple, because $S_{L}$ consists of exactly one point. But when $p=1$, the situation is quite different: $S_{L}$ does not generally consist of one point. It may be empty, or a singleton, or an infinite set (cf. [1]).

In [2] deLeeuw and Rudin studied the set $S_{L}$, and determined the structure of $S_{L}$ in some restricted cases. An element $f \in H^{1}$ is called an exposed point of the unit ball of $H^{1}$ if $S_{L}=\{f\}$ for some $L=L_{\phi}$ with $\phi \in L^{\infty}$. In [2], a function $f$ in $H^{1}$ is called strong outer if $f$ is not divisible in $H^{1}$ by any function of the form $(a-z)^{2}$ with $\alpha \in T$. It was proved that, in the restricted case they considered, $f$ is an exposed point if and only if $f$ is a strong outer function of norm 1. It is natural to ask whether this result can be extended to more general cases.

In [3] E. Hayashi presented examples of strong outer functions of norm 1 which are not exposed. His examples are of the form $(1-q(z))^{2}$ for some nonconstant inner

*This research was partially supported by Grant-in-Aid for Scientific Research, Ministry of Education. 
function $q(z)$, and, in [7], it is conjectured that $f \in H^{1}$ of norm 1 is exposed if and only if $f$ is not divisible in $H^{1}$ by any function of the form $(1-q(z))^{2}$ with a nonconstant inner function $q(z)$.

The purpose of this paper is to present a counterexample to the conjecture in [7] stated above. Our example is related to an infinite product of outer functions, which takes non-negative boundary values a. e. on $T=\partial D$. For the problem of characterizing exposed points in $H^{1}$, we can consult papers such as [3], [4], [6] and [7].

\section{Construction of a Counterexample. Let}

$$
F(z)=\prod_{k=1}^{\infty} \frac{\left(z-a_{k}\right)\left(1-\overline{a_{k} z}\right)}{(z-1)(1-z)}(z \in D)
$$

where $a_{k}=e^{i / k^{2}}, k=1,2,3, \ldots$ Note that the right-hand infinite product in (1) converges on each compact set $K$ of $C \backslash\{1\}$, since we have

$$
\sup _{z \in K} \sum_{k=1}^{\infty}\left|1-\frac{z-a_{k}}{z-1}\right|+\left|1-\frac{1-\overline{a_{k} z}}{1-z}\right| \leqq \sup _{z \in K} \frac{1+|z|}{|z-1|} \sum_{k=1}^{\infty}\left|1-e^{i / k^{2}}\right|<\infty
$$

The following properties of $F(z)$ hold:

(i) $F(z)$ is analytic on $D$ and can be extended analytically across $T \backslash\{1\}$.

(ii) $F\left(e^{i t}\right) \geqq 0$ for each $e^{i t} \in T \backslash\{1\}$.

(iii) If $z \in \bar{D} \backslash\{1\}, F(z)=0$ if and only if $z=\alpha_{k}$, and $\alpha_{k}$ is a zero of order 2 of $F$ for $k=1,2, \ldots$

(iv) $F(z)$ is outer.

(i) (iii) follows from the standard properties of infinite products. To see (iv), we consider $\log F(z)$ on $\bar{D} \backslash\{1\}$ such that $\operatorname{Im}[\log F(-1)]=0$, where $\operatorname{Im} z$ means the imaginary part of the complex number $z$. Since $\operatorname{Im}\left[\log F\left(e^{i t}\right)\right]$ is a monotone decreasing step function on $(0,2 \pi)$ with jumps $-2 \pi$ at $t=1 / k^{2}(k=1,2, \ldots)$ by the properties (i), (ii) and (iii) of $F(z)$ above, one sees that $\operatorname{Im}[\log F]$ is a real harmonic function belonging to the class $L \log ^{+} L$. Therefore the harmonic conjugates of $\operatorname{Im}[\log F]$ are in

$$
h^{1}=\left\{u: \text { harmonic on } D, \sup _{0 \leqq r<1} \int_{0}^{2 \pi}\left|u\left(r e^{i t}\right)\right| d t<\infty\right\}
$$

(cf. [5]), which implies that $F(z)$ is outer.

Next, choose $\varepsilon_{k}>0$ so that

$$
\frac{1}{k^{2}}>\frac{1}{k^{2}}-\varepsilon_{k}>\frac{1}{(k+1)^{2}}+\varepsilon_{k+1},\left|F\left(e^{i t}\right)\right| \leqq 1\left(t \in\left(\frac{1}{k^{2}}-\varepsilon_{k}, \frac{1}{k^{2}}+\varepsilon_{k}\right)\right) k=1,2, \ldots
$$


and put

$$
\Omega=\bigcup_{k=1}^{\infty}\left(\frac{1}{k^{2}}-\varepsilon_{k}, \frac{1}{k^{2}}+\varepsilon_{k}\right)
$$

If we define a function $g\left(e^{i t}\right) \in L^{1}(T)$ by

$$
g\left(e^{i t}\right)=\left\{\begin{array}{l}
\min \left\{\frac{1}{\left|F\left(e^{i t}\right)\right|}, 1\right\}: t \in(0,2 \pi) \backslash \Omega \\
\frac{1}{\varepsilon_{k} k^{4}}: t \in\left(\frac{1}{k^{2}}-\varepsilon_{k}, \frac{1}{k^{2}}+\varepsilon_{k}\right), \quad k=1,2,3, \ldots,
\end{array}\right.
$$

$\log g\left(e^{i t}\right)$ belongs to $L^{1}(T)$. Indeed, we can see this from:

$$
\begin{aligned}
& \int_{0}^{2 \pi}\left|\log g\left(e^{i t}\right)\right| d t \\
& \quad \leqq \int_{(0,2 \pi) \backslash \Omega}|\log | F\left(e^{i t}\right)|| d t+\sum_{k=1}^{\infty} \int_{1 / k^{2}-\varepsilon_{k}}^{1 / k^{2}+\varepsilon_{k}}\left|\log \frac{1}{\varepsilon_{k} k^{4}}\right| d t \\
& \leqq\|\log |F|\|_{1}-2 \varepsilon_{1} \log \varepsilon_{1}+\sum_{k=2}^{\infty} 2 \varepsilon_{k}\left(\log \frac{1}{\varepsilon_{k}}+\left|\log \frac{1}{k^{2}}\right|\right) \\
& \quad \leqq\|\log |F|\|_{1}-2 \varepsilon_{1} \log \varepsilon_{1}+\sum_{k=2}^{\infty} \frac{4}{k^{2}}\left|\log \frac{1}{k^{2}}\right|<\infty
\end{aligned}
$$

since $1 / k^{2}>\varepsilon_{k}$ by definition and $x \log 1 / x$ is an increasing function of $x$ on $(0,1 / e)$.

Using $g\left(e^{i t}\right)$, we define an outer function $f(z) \in H^{1}(D)$ by

$$
f(z)=\lambda \exp \int_{0}^{2 \pi} \frac{e^{i t}+z}{e^{i t}-z} \log g\left(e^{i t}\right) d t / 2 \pi(z \in D),
$$

where $\lambda$ is a positive constant to assure $\|f\|_{1}=1$. Then $f(z)$ has the following properties:

(a) For each $\delta(2>\delta>0)$, inf $\{|f(z)|: z \in D,|z-1| \geqq \delta\}>0$,

(b) $f(z) /(1-q(z))^{2} \notin H^{1}(D)$ for each non-constant inner function $q(z)$,

(c) $f(z)$ is not an exposed point of the unit ball of $H^{1}(D)$.

Proof of (a). We choose a continuous function $h\left(e^{i t}\right)$ on $T \backslash\{1\}$ such that $0<h\left(e^{i t}\right) \leqq$ $\lambda g\left(e^{i t}\right)(t \in(0,2 \pi))$ with $\log h \in L^{1}(T)$, and let $H$ the harmonic extension of $\log h$ to $\bar{D} \backslash\{1\}$. 
Since $\log |f(z)|$ and $H(z)$ are the Poisson integrals of $\log \lambda g$ and $\log h$ respectively, we get for each $\delta(0<\delta<2)$

$$
\inf \{|f(z)|: z \in D,|z-1| \geqq \delta\} \geqq \inf \{\exp H(z): z \in \bar{D},|z-1| \geqq \delta\}>0 .
$$

Proof of (b). First, we show that

$$
\frac{f(z)}{(1-a z)^{2}} \notin H^{1}
$$

for each $a \in T$. By (a), we may assume $a=1$. Then

$$
\begin{aligned}
& \int_{0}^{\pi}\left|f\left(e^{i t}\right)\right| \frac{1}{\mid 1-e^{\left.i i\right|^{2}}} d t \geqq \int_{\Omega} \lambda g\left(e^{i t}\right) \frac{1}{\left|1-e^{i n}\right|^{2}} d t \\
& \quad=\sum_{k=1}^{\infty} \lambda \int_{1 / k^{2}-\varepsilon_{k}}^{1 / k^{2}+\varepsilon_{k}} \frac{1}{\varepsilon_{k} \cdot k^{4}} \cdot \frac{1}{4 \sin ^{2} t / 2} d t \\
& \geqq \lambda \sum_{k=1}^{\infty} \frac{1}{\varepsilon_{k} \cdot k^{4}} \cdot \frac{1}{4 \sin ^{2} k^{-2}} \cdot 2 \varepsilon_{k}=\lambda \sum_{k=1}^{\infty} \frac{1}{2}\left(\frac{k^{-2}}{\sin k^{-2}}\right)^{2}=\infty
\end{aligned}
$$

and hence $f(z) /(1-z)^{2} \notin H^{1}$.

Next, we consider the general case. Suppose that $f(z) /(1-q(z))^{2} \in H^{1}$ for an inner function $q(z)$. Then $(-q(z)) /(1-q(z))^{2}$ is non-negative on a. e. on $T$, and belongs locally to $H^{1}$ at every point of $T \backslash\{1\}$ by $(a)$. Therefore, $(-q(z)) /(1-q(z))^{2}$ can be extended analytically beyond every point of $T \backslash\{1\}$ by the Schwarz reflection principle, and hence the singular support of $q(z)$ can exist only at $z=1$. If $q(z) \neq z$ and $\neq$ constant, $1-q(z)$ takes zeros at some points of $T \backslash\{1\}$, and hence $f(z) /(1-q(z))^{2} \notin H^{1}(D)$, contradicting the assumption above. Therefore, if we recall that $f(z) /(1-z)^{2} \notin H^{1}$ which we proved above, the only possibility is $q(z)=$ constant, which implies that (b) holds.

Proof of (c). Since

$$
\left|f\left(e^{i t}\right) F\left(e^{i t}\right)\right| \leqq\left\{\begin{array}{l}
\lambda: t \in \Omega \\
\frac{\lambda}{\varepsilon_{k} \cdot k^{4}}: t \in\left(\frac{1}{k^{2}}-\varepsilon_{k}, \frac{1}{k^{2}}+\varepsilon_{k}\right) k=1,2, \ldots
\end{array}\right.
$$

we get

$$
\int_{0}^{2 \pi}\left|f\left(e^{i t}\right) \cdot F\left(e^{i t}\right)\right| d t \leqq 2 \pi \lambda+\sum_{k=1}^{\infty} \frac{\lambda}{\varepsilon_{k} \cdot k^{4}} \cdot 2 \varepsilon_{k}<\infty
$$


Thus, $f(z) F(z) /\|f(z) F(z)\|_{1}$ is in the boundary of the unit ball of $H^{1}$ and has the same argument as $f(z)$ at almost every point of $T$, and hence $f(z)$ is not an exposed point of the unit ball of $H^{1}$.

The author would like to express his thanks to the referee for careful reading and valuable suggestions.

\section{REFERENCES}

1. P. L. Duren, Theory of $H^{p}$ spaces (Academic Press Inc., New York and London 1970).

2. K. DELeEuw and W. Rudin, Extreme points and extremum problems in $H_{l}$, Pacific J. Math. 8 (1958), 467-485.

3. E. Hayashl, Solution sets of extremal problems in $H^{l}$, Proc. Amer. Math. Soc. 93 (1985), 690-696.

4. H. Helson, Large analytic functions II, in Analysis and Partial Differential Equations (ed. Cora Sadosky, Marcel Dekker, 1990), 217-220.

5. P. Koosis, Introduction of $H^{1}$ spaces (Cambridge University Press, 1980).

6. T. Nakazl, Sum of two inner functions and exposed points in $H^{1}$, Proc. Edinburgh Math. Soc. 35 (1992), 349-357.

7. D. Sarason, Exposed points in $H^{1}, 1$, Oper. Theory Adv. Appl. 41 (1989), 485-496.

Department of Mathematics

HOKKAIDO UNIVERSITY

SAPPORO 060

JAPAN 